\title{
Shape Abstraction Using Computational Topology Techniques
}

\author{
S. Biasotti, B. Falcidieno, M. Spagnuolo \\ Istitituto per la Matematica Applicata - CNR
}

\begin{abstract}
This paper investigates the possible role of the new field of computational topology for incorporating abstraction mechanisms in shape modelling. The effectiveness of computational topology techniques is exemplified with an application of discrete differential topology. In particular, a method is proposed for the extraction of a critical point configuration graph from a triangulated surface. Starting from the definition of the Reeb graph in the smooth domain, the concept of critical point is extended to critical areas, which may represent isolated as well as degenerated critical points in the discrete domain. The resulting graph effectively represents the surface shape and has been successfully used as a basis for model compression and restoring purposes.
\end{abstract}

Key words: Computational topology, shape graph, discrete critical point analysis.

\section{INTRODUCTION}

Geometric modelling deals with the representation and manipulation of geometric objects in a computer and its foundations are set by the definition of formal properties which can be used to completely describe the shape of objects. Even if shape is surely characterised by a specific geometry, shape information is treated differently by the human brain to many other forms of information. Also experiments in human perception suggest that people use different models for shape interpretation, both high (specific) and low (generic) level, which are constructed using operations such as: grouping surface portions having a similar shape, identifying features, abstracting the 
shape and then synthesising it using descriptive labels related to the semantic context (Falcidieno\&Spagnuolo, 1998).

Topology as the study of shape properties that do not change under deformation, gives a formal framework for the formalisation and solution of several problems related to shape and shape understanding. Since the beginning, the importance of topological aspects in modelling and analysis has been recognised in many computer application areas. Lately, this has caused the starting of this new field which has been called computational topology, term firstly introduced and described in (Dey et al., 1998) and followed by authors such as (Hart, 1999; Axen\&Edelsbrunner 1998).

When using structured geometric models, such as the CSG or B-rep models, several shape features and properties can be easily associated to groups of entities defined by topological and geometric relationships. Conversely, in many application areas we have to deal with complex models whose geometry is more and more frequently represented by triangular meshes. Triangular meshes are very flexible structures and can be used both in design and reconstruction processes, but they do not offer any partial structuring of the geometry which can be used to deduce global shape information. Consequently, there has been a growing interest and great research effort put in several problems related to the use of triangular meshes. These were, for example, the simplification and compression, or the optimisation and re-meshing for getting so-called quality meshes. In this context, it is interesting to investigate the possible role of computational topology for incorporating abstraction mechanisms in shape modelling.

From a mathematical point of view, polyhedral meshes have been topic of research in general topology since decades and have formed a natural foundation for the development of algebraic topology, initially known as combinatorial topology (Engelking\&Sielucki, 1992). Here, topological properties of the polyhedra, such as the Betti numbers and the related and well-known Euler equation, can be simply computed by counting the basic elements (simplices) of the polyhedra. These concepts have been widely used in solid modelling to approach problems related to the validity of complex data structures, to the extraction of features from models or to the reconstruction of surfaces from discrete data set.

Among the different domains related to computational topology, this paper deals with some aspects of differential topology, which has been often used for surface coding and description. In this context, Morse theory sets the foundations for associating the topology of a given manifold to the critical points of a smooth function defined on the manifold. Starting from Morse theory, it is possible to define a graph, the Reeb graph, which represents a smooth surface by coding the evolution of its contours and which is particularly interesting for shape understanding and compression 
(Reeb, 1946; Shinagawa et al., 1991a). Moreover, the juxtaposition of critical points on a surface is generally considered as one of the simplest way in which surface shape is perceived and organised (Pentland, 1986). The configuration of critical points has been used for shape description (Nackman, 1984; Pfaltz, 1990) as well as for driving the simplification of meshes while preserving the topology of a shape (Bajaj\&Schikore, 1998).

Using its original formulation, the Reeb graph is considered and adapted to handle triangular meshes with the aim of defining a shape analysis tool for triangular meshes. In particular, we propose the use of critical areas instead of critical points and extend the domain of applicability of the Reeb graph description to surfaces that do not belong to the Morse class. The set of possible relations among critical areas is also extended. In section 2, the basic concepts of Morse theory and Reeb graph are presented in the smooth domain, while the approach adopted to formulate a similar shape description in the discrete domain is given in section 3. An algorithm for computing the graph is briefly described in section 4 , where the application to surface compression and restoring is also sketched. Conclusions are drawn in the final section.

\section{CRITICAL POINTS OF SMOOTH FUNCTIONS: MORSE THEORY AND REEB GRAPHS}

Since its introduction, Morse theory has been considered as a fundamental tool for analysing the topology of smooth manifolds. The basic idea is that the topology of a given manifold can be described by analysing the critical points of a smooth function defined on the manifold. The simplest example of this relationship is the following well-known result: if a manifold is compact, then any continuous function defined on it has a maximum and a minimum. In applications related to geometric modelling, it is quite natural to choose the height function to study the surface shape. Intuitively, the height function of a smooth manifold $M$, embedded into the usual three-dimensional Euclidean space, is the real function, which associates its elevation to each point on the surface. The critical points of the height function will correspond to locations where the tangent plane is horizontal, while the level sets correspond to the intersections of the surface with planes orthogonal to the height direction.

The height function, and in general any real smooth function $f$, defined on a smooth manifold $M$, is called Morse function if all of its critical points are non-degenerate. A critical point is non-degenerate if the Hessian matrix $H$ of $f$ is non-singular at that point. In particular non-degenerate critical points are 
isolated, therefore plateaux or volcano rims do not comply with the definition of Morse function (Milnor, 1963).

An example of a simple Morse function is given in figure 1(a), while figure 1(b) depicts an example of a degenerate critical point, a so called "quadriped-saddle"; finally figure 1(c) shows an example of non isolated critical points.

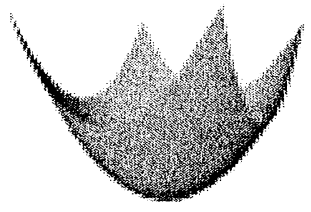

(a)

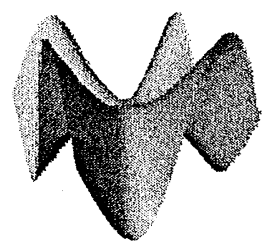

(b)

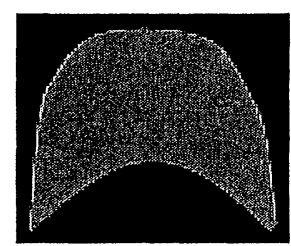

(c)

Figure 1. Examples of critical points: a simple minimum (a), a $4^{\text {th }}$ order saddle (b) and a volcano rim (c).

In general, the level sets may be shaped in arbitrarily complex ways, but if the height function is Morse, then their configuration can be quite simply classified and the associated manifold can be decomposed into a limited set of primitive topological cells (Milnor, 1963). Applications of this theory in computational contexts can be found, for example, in (Nackmann, 1984) where the shape of Morse functions is described by classifying the possible cycles occurring in the critical point configuration graph. These cycles correspond to regions of uniform behaviour of the first derivative, the socalled slope districts, and have been classified into ten basic types.

Using a similar approach, the shape of a manifold can be effectively represented using the Reeb graph, defined to code the evolution and arrangement of level curves (Reeb, 1946; Shinagawa et al., 1991a). The Reeb graph of a real valued function $f$ is defined as follows:

Definition: Let $f: M \rightarrow \Re$ be a real valued function on a compact manifold $M$. The Reeb graph of $M$ wrt $f$ is the quotient space of $M x \Re$ defined by the equivalence relation " $\sim$ ", given by:

$\left(X_{1}, f\left(X_{1}\right)\right) \sim\left(X_{2}, f\left(X_{2}\right)\right) \Leftrightarrow f\left(X_{1}\right)=f\left(X_{2}\right)$ and $X_{1}$ and $X_{2}$ are in the same connected component of $f^{-1}\left(f\left(X_{1}\right)\right)$

Therefore, all points of a compact manifold having the same value under a real function and whose pre-image belongs to the same connected component are collapsed into one element (see figure 2(a)). Moreover, since the contour topology changes only at critical levels of the height function, it is possible to associate a graph structure to the Reeb's quotient space, as follows. First of all, the node set is defined by those equivalence classes in the Reeb's quotient space, which correspond to critical levels of the height function. Then, an arc is defined by identifying all the equivalence classes 
resulting from contours between two critical levels and belonging to the same connected component (Milnor, 1963; Biasotti et al., 2000a).

In figure 2(a) the equivalence classes defined over the height function of a bi-torus are shown. In figure 2(b) the Reeb's quotient space is represented as a "traditional" graph where the equivalence classes are grouped into arcs if they are representative of diffeomorfic contours, as previously stated.

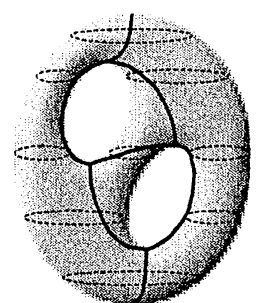

(a)

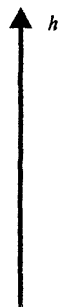

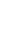

Figure 2. The Reeb graph of a bi-torus wrt the height function h: the equivalence classes are represented by the black solid line on the surface (a), and the corresponding graph representation is depicted in (b).

Obviously, since the choice of the mapping function is not unique, a manifold can admit different Reeb graphs. However, if we have to describe the shape of a manifold surface $M$ embedded into the Euclidean space, the Reeb graph of $M$ under its "natural" height function codes the shape in terms of meaningful semantic labels, such as peaks, pits or passes. Moreover, under the assumption that the height function is Morse, the structure of the Reeb graph is rather simple: the nodes have almost degree three and the arcs of the graph can be oriented.

This property is a consequence of a fundamental result of Morse theory, which establishes a link between the number of critical points and the topological type of the underlying manifold. Indeed, a Morse function defined on two-manifold satisfies the following Euler formula:

$$
\text { maxima }- \text { saddles }+ \text { minima }=2(1-g)=\chi(M)
$$

where $g$ represents the genus of the manifold (Milnor, 1963; Griffiths, 1976). The value $\chi(M)$ is called the Euler's characteristic of the manifold $M$, and is related to the manifold Betti numbers.

The use of Reeb graphs in computer graphics has been firstly addressed in (Shinagawa et al., 1991a; Shinagawa\&Kunii, 1991b). Even if there are no restrictions in the Reeb graph definition on the type of the function $f$ associated to the manifold, several authors have in practice limited the use of Reeb graphs to Morse mapping functions. In the following section, it will be shown how a structure similar to the Reeb graph can be defined in the 
discrete domain, starting from a triangular mesh and considering critical areas instead of critical points.

\section{CRITICAL AREAS OF TRIANGULAR MESHES}

The theories deriving by the adaptation of smooth techniques to discrete domains are often surprising, as many differential topology and geometry results have combinatorial analogues (Forman, 1993). In strictly mathematical formulations, the discrete Morse theory has been approached using slightly different approaches. Here, we are mainly interested in coding the shape of a triangular mesh in a topological structure defined using the Reeb graph idea, and which keeps the same meaning as its smooth counterpart.

First of all, let us restrict the context of the discussion to triangulated scalar fields, i.e. surfaces defined by function such as $z=f(x, y)$; the extension to the three dimensional case will be discussed in the conclusive section. A formal and complete definition of critical points on a discrete mesh can be found in (Banchoff, 1970; Forman, 1993), where critical points are defined by taking into account the difference in elevation among a point and its star-neighbours (see figure 3). A simplified version of the critical point classification scheme has been used for example in (Bajaj\&Schikore, 1998; Takahashi et al., 1995) for constructing topological structures to represent a given mesh. These approaches, anyway, do not consider all the possible configuration of edges around a point, and, for example, saddle points having more than two ascending directions, (see figure 3) are not considered. Thus, separating such saddle points in simpler ones a non-unique interpretation is introduced.
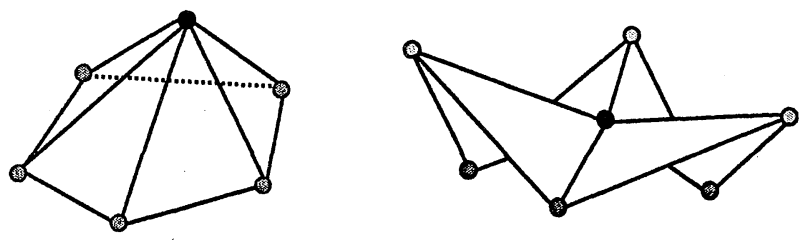

Figure 3. The configuration of neighbours around a point which give rise to a maximum (a), and to a saddle in (b).

Critical points can be detected also by studying the evolution of level sets, or contours, defined by intersecting the surface with a plane orthogonal to the height direction. The configuration and containment relationship among contours allows us to find critical points: for example, a contour 
which does not contain any other contour will locate a maximum or a minimum point (Milnor, 1963; Lipschutz, 1969; Aumann et al. 1991). Studying the evolution of contours provides a more global view of the surface shape with respect to local neighbourhood computation. Moreover, the frequency of the contours, i.e. their distance, behaves as a filter for discarding small ondulations which would cause the detection of many irrelevant critical points. Contours have been used also in (Shinagawa et al., 1991a; Shinagawa\&Kunii, 1991b) for analysing smooth surfaces with a Morse height function. Given a generic continuous mesh, our approach is to compute a dense number of contour lines, to analyse the resulting critical areas, and finally to construct an extended Reeb graph representation from the topological adjacency relationship among critical areas. To correlate the distance between contours to the feature size, we have chosen to use the minimum vertical distance among vertices as a reference value.

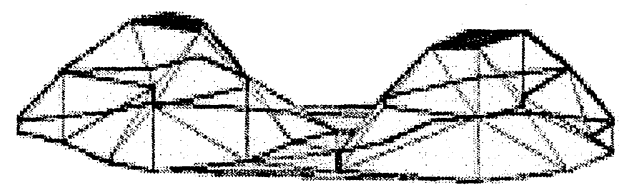

Figure 4. Critical areas defined on a triangulation constrained to contours.

Therefore, the pre-image of any critical level of the height function associated to $M$ will be, in general, represented by a level region of $C_{T}(M)$. These regions are defined as the critical areas of $M$.

Since we do not require the height function to be Morse, the flat regions of $\mathrm{C}_{\mathrm{T}}(\mathrm{M})$ correspond either to simply or to multiply connected areas, which define, respectively, simple or complex critical areas. Simply connected critical areas may correspond either to isolated or degenerate critical points of M. A multiply connected critical area corresponds to a degenerate critical level, and it divides the surface into two parts: an outer part defined by the surface not contained in the area, and as many inner parts as the multiplicity of the boundary. The critical areas are firstly classified as simple or complex and then each element is further classified as minimum, maximum or saddle according to the type of boundary edges and to the behaviour of the height function across the boundary. The complete classification scheme is presented in (Biasotti et al., 2000a).

In figure 5, a surface is depicted with its contour levels (a), and the classified critical areas (b). Simple maximum and minimum areas are depicted in dark grey and saddles in light grey; the complex maximum is depicted in grey. 


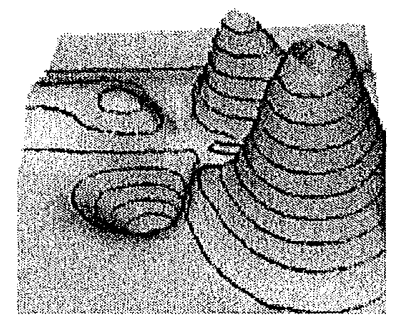

(a)

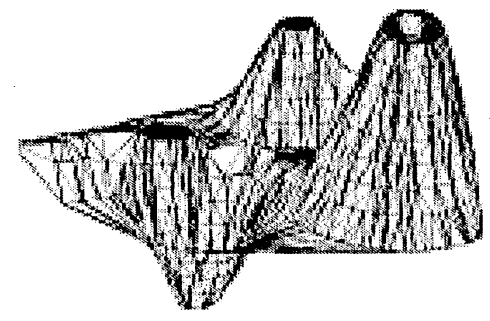

(b)

Figure 5: Critical areas classification: the initial contour set (a) and the critical areas: simple maximum and minimum areas are coloured in dark grey, simple saddles in light grey and the complex maximum in medium grey (b).

Since we are considering surfaces with boundary, it is necessary to give a unique interpretation to those critical areas touching the boundary. This is easily accomplished by considering a global virtual minimum point, which does not correspond to any real point in space, and which has the aim of assigning descending directions to all edges on the surface boundary (Takahashi et al. 1995).

Obviously, not all flat regions of $C_{T}(M)$ correspond to critical points of the height function: ridges and ravines also produce flat zones, which can be anyway easily detected checking the number of ascending or descending directions out of the zone boundary. Details can be found in (Biasotti et al. 2000a; Auman et al. 1991, De Martino\&Ferrino, 1996).

It is interesting to briefly discuss the validity of the Euler formula for the defined critical areas. With the introduction of the virtual minimum, the Euler formula is still satisfied, if the critical areas correspond to critical points, isolated or not, but with multiplicity equal to one (i.e. critical points such as the quadriped-saddle in fig. 1(b) are not allowed). First of all, let us observe that the virtual minimum guarantees the topological equivalence between the surface and a 2-sphere. A generalised Euler formula can be introduced which takes into account the number of simple as well as complex critical areas. For each complex critical area, $c_{a}$, let us call $h$ the number internal components of $c_{a}$ and indicate $m c_{a}=h-1$. Let be $P_{m c}$ the sum of all $m c_{a}$ values for each complex area, that is, $P_{m c}=\sum_{c_{a}} m c_{a}$. Then, our version of the Euler formula for triangulated surfaces becomes:

$$
\text { maxima - saddles }+ \text { minima }-P_{m c}+m v=2
$$

where $m v$ is the contribution due to the virtual minimum.

To explain the meaning of the value $m c_{a}$ introduced above and to intuitively justify the consistency of the generalised Euler formula in (2) with the formulation given in (1), let us consider the example depicted in 
figure 6. Here, the highlighted complex maximum represents a critical level composed of non-isolated maxima of the height function. The complex maximum has only one inner component, and thus $h=1$ and $m c_{a}=0$. Therefore, its contribution to the extended Euler's formula is 0 . Let us now consider a small rotation of the surface: this would cause the degenerate critical level to be decomposed into one maximum and one saddle, as shown in figure 6(b), and the contribution of this configuration to the Euler formula would be again 0 . Similarly, if we consider a complex maximum having two inner components $\left(h=2, m c_{a}=1\right)$ then the contribution to the Euler formula is -1 . If we let the surface slightly rotate, then the critical point configuration would be made of two saddles and one maximum, and again, its contribution to the extended Euler equation is -1 .

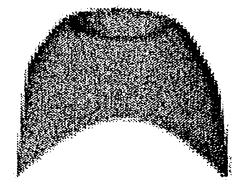

(a)

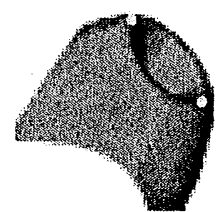

(b)

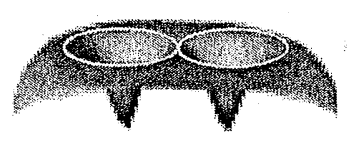

(c)

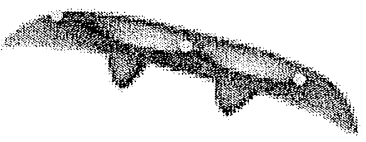

(d)

Figure 6: The circle in (a), totally composed of maximum points, is transformed by rotation into a maximum and a saddle point (b). Similarly, a complex maximum having two cycles and two inner components (c), is decomposed into two saddles and one maximum (d).

Another way of justifying the Euler equation is to consider the relation between critical areas and Reeb graph nodes. By definition, all points belonging to a simply connected critical area are Reeb-equivalent and may therefore collapse into the same node. If the isolated critical points of $M$ were known, moreover, a simple labelling of the graph's node set would be sufficient to distinguish them from degenerate critical points. Similarly, the behaviour of arcs incident to simple critical areas is equivalent to the behaviour of arcs incident to isolated critical points. Therefore, simple critical areas can be represented in an extended Reeb graph model by simple nodes as in the normal Reeb graph representation. Multiply connected critical areas correspond to macro-nodes: that is particular nodes having at least one arc connected to an inner node. Note that the equation in 2 can be easily extended to closed surfaces, by considering an analogous definition of critical area (Biasotti, 2000c). 


\section{CONSTRUCTION OF THE REEB GRAPH}

In this section we will briefly sketch an algorithm to automatically construct the Reeb graph of a triangulation. The resulting shape representation is called Extended Reeb Graph (ERG) and is defined as a graph whose nodes correspond to simple critical areas, macronodes correspond to complex critical areas, and arcs represent the topological adjacency among critical areas.

In the following, we will assume that the input is defined by a contour set, either given or computed. From an algorithmic point of view, the first step is the construction of a Delaunay triangulation constrained to the contours and the recognition of the critical areas, while the node created in correspondence to the virtual minimum, $V M$, initialises the ERG. Such a node does not correspond to any real point in the space, and we visualize it as a point having elevation smaller than any other point on the surface. The critical areas are detected by extracting the flat areas of the constrained Delaunay triangulation and the classification of critical areas, simple or complex, is done by checking the number of non-constrained edges in the boundary, as described in (Biasotti et al., 2000a).

To extract the arcs of the ERG, we have introduced the concept of influence zone, which is defined using the edge-adjacency relationship among contours; intuitively the influence zone of a given critical area spans the part of surface topologically adjacent to that area. In practice, influence zones for minimum and maximum areas are computed using an expansion process that connects diffeomorfic contours located between two critical levels (cfr. Section 2). Consequently, influence zones of maxima and minima are disjoint and cannot overlap. Influence zones of saddle areas delimit the surface portion where the topological change occurs. Therefore, saddle zones having the same elevation and being topologically adjacent, may have the same influence zone. In this case, the influence zone is unique for the saddles, and it will correspond to the same node in the ERG.

All influence zones of the surface in figure 3 are depicted in figure 7(a).

Then, using the notion of influence zone, a first set of ERG arcs is extracted from the adjacency relationships between the influence zones of simple maximum or minimum critical areas and saddle ones. In this manner the arcs connected to terminal nodes of the $E R G$ are identified (see figure 7(b)). To complete the $E R G$ construction the links between saddles and, in general, complex areas have to be determined. Intuitively, arcs correspond to ascending paths between critical areas, which are determined by expanding the associated influence zones, following free directions in the outmost boundary component (see figure $7(\mathrm{c})$ ). Free directions are those which do not correspond to an already identified arc. 


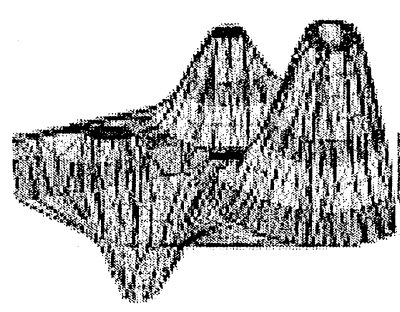

(a)

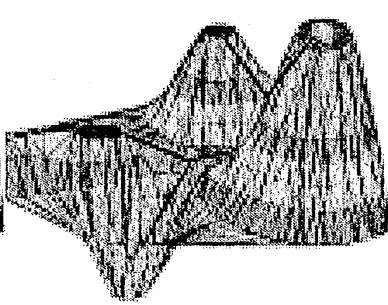

(b)

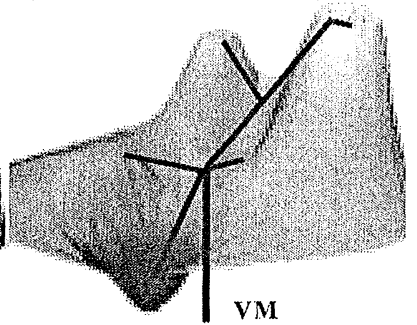

(c)

Figure 7: The influence zones (a), the first step of the ERG construction (b) and the final one (c). The virtual minimum is connected to the saddle at minimum elevation.

In figure 8, an example of our characterisation method applied to a natural surface is shown. In (a) the initial surface is depicted with its contour lines in (b). Here, the critical areas are depicted in dark grey. The resulting ERG representation is shown in (c).

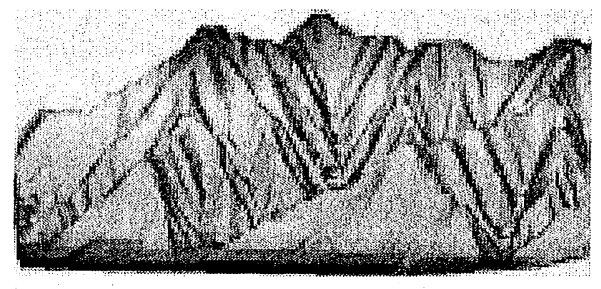

(a)

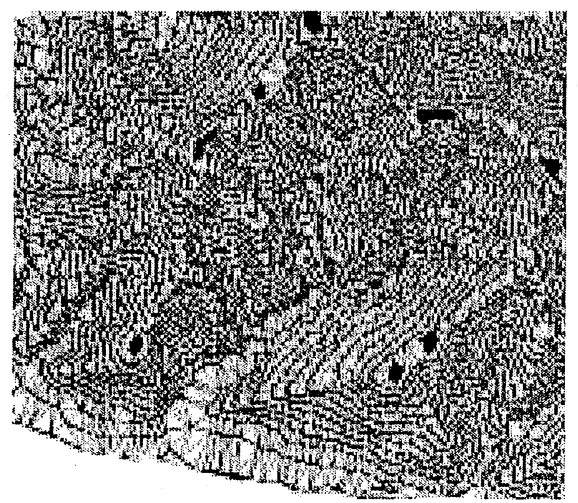

(b)

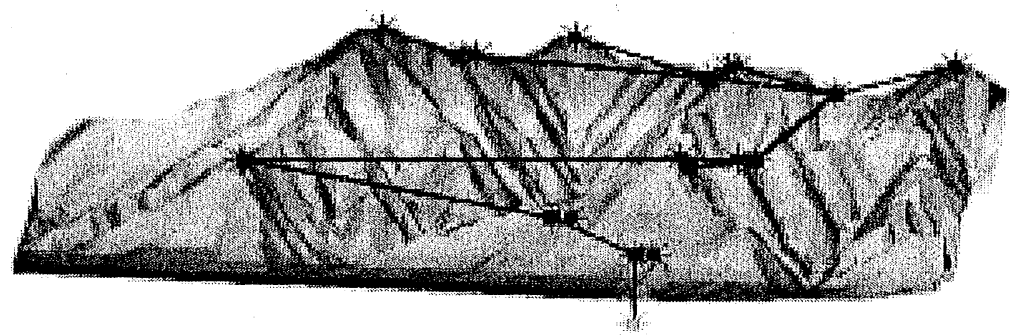

(c)

Figure 8: A mesh representing a terrain (a); the characterized model (b) and the resulting ERG representation (c).

The ERG has been used for compression and decompression of triangular meshes (Biasotti et al., 2000b). The compressed model can be simply constructed by triangulating the critical levels identified by the ERG nodes. 
The decompression step is aimed at associating appropriate restoring rules to the ERG, so that the reconstruction of the original shape from the graph can be done automatically. This problem is similar to surface reconstruction from cross section. In this case, however, the relationships stored in the ERG simplify the branching problem, but on the other side the sections are very sparse. Therefore, tools are needed to blend correctly the shape of one contour into the adjacent one, generating sufficiently dense intermediate contours among critical sections. As well as our approach to model compression is based on the shape analysis, also the approach to contour blending is based on the analysis of shape similarities between contours (Mortara\&Spagnuolo, 2000). To summarize, the proposed approach to model simplification is strongly based on the comprehension of the surface shape, therefore using a top-down approach to model compression. In this case, data reduction is seen not simply as a matter of "quantity" but of "quality": a model may result too bulky for a specific application not only because it "contains too many points" but mainly because it is defined at a too low abstraction level.

\section{CONCLUSIONS}

The combination of geometry and topology provides interesting insight into several computer application problems that involve shape modeling and processing. Computational approaches to topological questions are increasing and we might foresee that computational topology will eventually develop into an area of computer science.

In this context, we have described a new method for analyzing the shape of triangular meshes, based on an extended Reeb graph representation. The result is a high-level description of the surface shape, which provides a model compression mechanism of really great interest.

We have shown examples produced with our first implementation. The natural next step is the extension of the method to handle fully 3D input. To this aim we are extending both the critical area classification and the ERG representation algorithm to three-dimensional surfaces without boundary (Biasotti, 2000c; Attene et al., 2001). From the first results we can affirm that the presented shape classification is still valid for closed surfaces. In fact, starting from a Delaunay triangulation constrained to level sets; flat areas still correspond to surface's critical points. Also, influence zones can be defined analogously to the bi-dimensional case. The graph extraction algorithm differs from the bi-dimensional version because the virtual minimum introduction is not necessary in this case: for closed surfaces the construction process starts from the critical area at minimum elevation. 
Moreover, for closed surfaces, cycles may appear in the ERG, due to surface holes. Finally, in figure 9 we present an example of ERG extraction for a 3D closed surface.

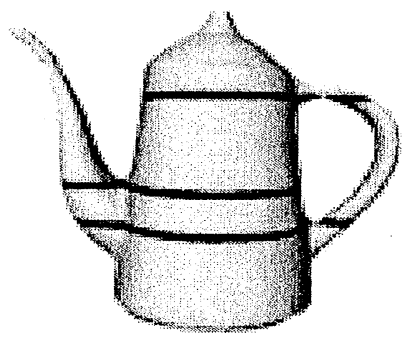

(a)

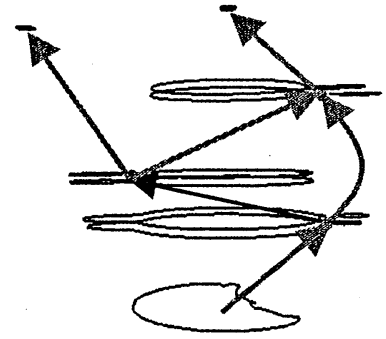

(b)

Figure 9: The influence saddle zones of a 3D model (a) and the graph representation obtained considerinfg only the critical sections (b).

\section{REFERENCES}

Attene, M., Biasotti, S., Spagnuolo, M. (2001). Re-meshing Technique for Topologycal Analysis. In Proceedings of Shape Modelling International '01, Genova, Italy, May 2001.

Aumann, G., Ebner, H., Tang, L. (1991). Automatic derivation of skeleton lines from digitised contours. ISPRS Journal of Photogrammetry and Remote Sensing, 46.

Axen, U., Edelsbrunner, H. (1998). Auditory Morse Analysis of Triangulated Manifolds. Mathematical Visualization, Springer Verlag.

Bajaj, C., Schikore, D. R. (1998). Topology preserving data simplification with error bounds. Computer \& Graphics, 22(1).

Biasotti,S., Falcidieno, B., Spagnuolo, M. (2000a). Extended Reeb Graphs for Surface Understanding and Description. Proceedings of $9^{\text {th }}$ Discrete Geometry for Computer Imagery conference, LCNS 1953, Springer Verlag, Uppsala.

Biasotti, S., Mortara, M., Spagnuolo, M. (2000b). Surface Compression and Reconstruction using Reeb graphs and Shape Analysis. In Proceedings of the Spring Conference on Computer Graphics, Bratislava.

Biasotti, S. (2000c). Estensione dell'algoritmo di estrazione dei grafi di Reeb a superfici tridimensionali. Technical report IMA n ${ }^{\circ} 14 / 00$.

De Martino, M., Ferrino, M. (1996). An example of automated shape analysis to solve human perception problems in anthropology. International Journal of Shape Modeling, (21.

Dey, T. K., Edelsbrunner, H., Guha, S. (1999). Computational Topology. In Advances in Discrete and Computational Geometry, eds: Chazelle, B., Goodman, J. E., Pollack, R., Contemporary Mathematics 223, AMS, Providence.

Engelking, R. and Sielucki, K., (1992) Topology: a geometric approach. Sigma series in Pure Mathematics, Volume 4, Heldermann Verlag, Berlin, 1992.

Falcidieno, B., Spagnuolo, M. (1998). Shape Abstraction Paradigm for Modelling Geometry and Semantics. In Proceedings of Computer Graphics International, Hannover.

Fomenko, A.(1994). Visual Geometry and Topology. Springer-Verlag.

Forman, R. (1993) Morse theory for cell complexes. Manuscript, Department of Mathematics, Rice University, Houston, Texas. 
Griffiths, H. B. (1976). Surfaces. Cambridge University Press, $2^{\text {nd }}$ ed.

Hart, J.,C. (1999). Computational Topology for Shape Modelling. In Proceedings of Shape Modelling International '99, Univ. Aizu, Japan

Milnor, J. (1963). Morse Theory. Princeton University Press, New Jersey.

Mortara, M., Spagnuolo, M. (2000). Similarity measures for blending polygonal shapes. Computers \& Graphics, Special Issue on Shape Blending.

Nackman, L. R. (1984). Two-dimensional Critical Point Configuration Graphs. IEEE Transactions on Pattern Analysis and Machine Intelligence, Vol. PAMI-6, No. 4.

Pentland, P. (1986). Perceptual organization and representation of natural form. Artificial Intelligence, Vol.28.

Pfaltz, J. L. (1990). Surface Networks. Geographical Analysis, Vol. 8.

Reeb, G. (1946). Sur les points singuliers d'une forme de Pfaff completement integrable ou d'une fonction numèrique. Comptes Rendus Acad. Sciences, Paris, 222.

Shinagawa, Y., Kunii, T. L., Kergosien, Y. L. (1991a). Surface Coding Based on Morse Theory. IEEE Computer Graphics \& Applications.

Shinagawa, Y., Kunii, T. L. (1991b). Constructing a Reeb graph automatically from cross sections. IEEE Computer Graphics and Applications, 11(6).

Takahashi, S., Ikeda, T., Shinagawa, Y., Kunii, T. L., Ueda, M. (1995). Algorithms for Extracting Correct Critical Points and Construction Topological Graphs from Discrete geographical Elevation Data. Eurographics '95, Vol. 14, No 3. 